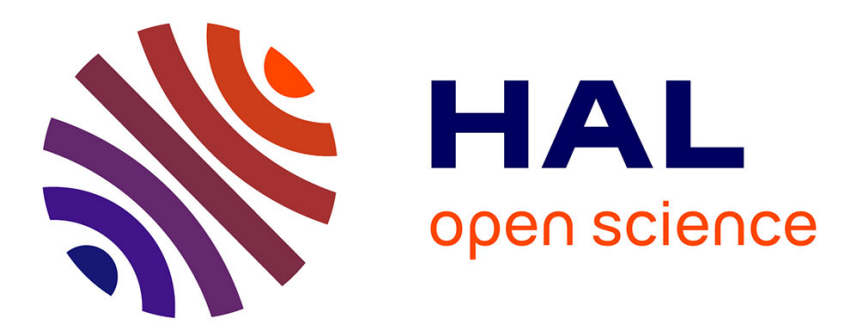

\title{
Dynamics of Turing and Faraday instabilities in a longitudinally modulated fiber-ring cavity
}

F. Copie, Matteo Conforti, Alexandre Kudlinski, Stefano Trillo, Arnaud Mussot

\section{- To cite this version:}

F. Copie, Matteo Conforti, Alexandre Kudlinski, Stefano Trillo, Arnaud Mussot. Dynamics of Turing and Faraday instabilities in a longitudinally modulated fiber-ring cavity. Optics Letters, 2017, 42 (3), pp.435-438. 10.1364/OL.42.000435 . hal-02386359

\section{HAL Id: hal-02386359 \\ https://hal.science/hal-02386359}

Submitted on 29 Nov 2019

HAL is a multi-disciplinary open access archive for the deposit and dissemination of scientific research documents, whether they are published or not. The documents may come from teaching and research institutions in France or abroad, or from public or private research centers.
L'archive ouverte pluridisciplinaire HAL, est destinée au dépôt et à la diffusion de documents scientifiques de niveau recherche, publiés ou non, émanant des établissements d'enseignement et de recherche français ou étrangers, des laboratoires publics ou privés. 


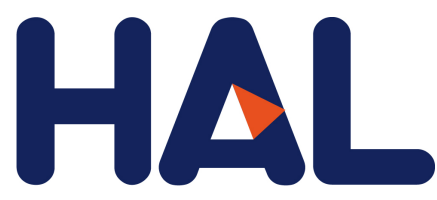

archives-ouvertes

\title{
Dynamics of Turing and Faraday instabilities in a longitudinally modulated fiber-ring cavity
}

\author{
F. Copie, M. Conforti, A. Kudlinski, S. Trillo, A. Mussot
}

\section{To cite this version:}

F. Copie, M. Conforti, A. Kudlinski, S. Trillo, A. Mussot. Dynamics of Turing and Faraday instabilities in a longitudinally modulated fiber-ring cavity. Optics Letters, Optical Society of America, 2017, 42

(3), pp.435. 10.1364/OL.42.000435 . hal-02386359

\section{HAL Id: hal-02386359 \\ https://hal.archives-ouvertes.fr/hal-02386359}

Submitted on 29 Nov 2019

HAL is a multi-disciplinary open access archive for the deposit and dissemination of scientific research documents, whether they are published or not. The documents may come from teaching and research institutions in France or abroad, or from public or private research centers.
L'archive ouverte pluridisciplinaire HAL, est destinée au dépôt et à la diffusion de documents scientifiques de niveau recherche, publiés ou non, émanant des établissements d'enseignement et de recherche français ou étrangers, des laboratoires publics ou privés. 


\title{
Dynamics of Turing and Faraday instabilities in a longitudinally modulated fiber ring cavity
}

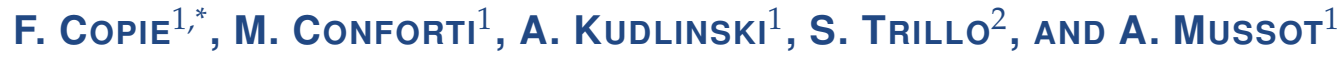 \\ ${ }^{1}$ Univ. Lille, CNRS, UMR 8523-PhLAM—Physique des Lasers Atomes et Molécules, F-59000 Lille, France \\ ${ }^{2}$ Department of Engineering, University of Ferrara, Via Saragat 1, 44122 Ferrara, Italy \\ *Corresponding author: francois.copie@univ-lille1.fr
}

Compiled December 20, 2016

\begin{abstract}
We experimentally investigate the roundtrip-toroundtrip dynamics of the modulation instability spectrum in a passive fiber $r$ ing $c$ avity $p$ resenting an inhomogeneous dispersion profile. B y implementing a real-time spectroscopy technique we are able to record successive single-shot spectra, which display the evolution of the system toward a stationary state. We find $t$ hat $t$ he $t$ wo i nstability $r$ egimes ( Turing and Faraday) that compete in this kind of inhomogeneous cavities not only differ by their characteristic frequency but also by their dynamical behaviour. The dynamic transition between those two regimes of instability is also presented.
\end{abstract}

OCIS codes: (190.4370) Nonlinear optics, fibers; (190.3100) Instabilities and chaos; (230.5750) Resonators

http://dx.doi.org/10.1364/ao.XX.XXXXXX

Modulation Instability (MI) is unarguably the most ubiquitous nonlinear phenomenon whether it be in hydrodynamics, plasma physics or optics [1]. In its best-known form it can be seen as a long-wave instability at the origin of the exponential growth of a periodic modulation on top of a homogeneous background solution in the time or the spatial domain. The signature in the frequency domain is the appearance of spectral sidebands symmetric to the pump frequency. MI also occurs in dissipative systems such as passive cavities where it is commonly referred to as the Turing instability, $[2,3]$. The latter has been extensively studied in the context of short pulse train generation in homogeneous cavities, where MI extends to the normal dispersion regime [4-8]. Recently, we showed that a longitudinal modulation of the dispersion inside the cavity can lead to the emergence of a new kind of coexisting parametric instability [9, 10], which is analogous to the Faraday instability occurring in a periodically forced system [11]. The Faraday or parametric instability is shared by optical resonators with modulation of other parameters as well $[12,13]$, and its universal nature is witnessed by observations in other fields [14]. Recent experiments showed that the Turing and Faraday unstable regimes can be independently excited and identified thanks to the different frequency of their characteristic sidebands in the optical spectrum. For a cer- tain range of parameters one can even switch from one instability regime to the other by tuning the input pump power [15]. In this previous work we limited our investigations to the stationary regime (i.e., spectra were recorded after a very large number of roundtrips thus ensuring that the system reached a stable state). Conversely, the development of real-time spectroscopy techniques such as the time-strech dispersive Fourier transformation (DFT) enabled the recording of shot-to-shot spectral fluctuations associated to ultrafast nonlinear phenomena such as MI [16, 17] or supercontinuum generation [18]. The recent years have seen a growing interest in this technique to investigate the roundtripto-roundtrip dynamics of both passive and active fiber cavities [19-22]. However, to date, experimental studies of the transient behaviour of $\mathrm{MI}$ in a passive ring resonator remain substantially unaddressed. In this Letter we experimentally investigate on the transient evolution of Turing and Faraday instabilities in a dispersion oscillating passive fiber cavity by implementing the DFT technique. We captured the growth and saturation of the power of the different unstable bands as well as the switching dynamics between Turing and Faraday instabilities by abruptly changing the input pump power. Our experimental results turn out to be well supported by numerical simulations of the cavity map model.

We conveniently describe the light propagation in the fiber ring cavity by the following Ikeda-like map for the intracavity field $E_{m}=E_{m}(z, \tau)$ in the $m$-th circulation $[9,10,23]$

$$
\begin{array}{r}
\frac{\partial E_{m}(z, \tau)}{\partial z}=\left(-\frac{\alpha_{f}}{2}-i \frac{\beta_{2}(z)}{2} \frac{\partial^{2}}{\partial \tau^{2}}+i \gamma\left|E_{m}(z, \tau)\right|^{2}\right) E_{m}(z, \tau) \\
E_{m+1}(0, \tau)=\rho E_{m}(L, \tau) \exp \left(i \phi_{0}\right)+\theta E_{i n}\left(\tau+m t_{R}\right)
\end{array}
$$

Equation (1) turns out to be the nonlinear Schrödinger equation which describes the propagation of the field during one roundtrip (i.e. from $z=0$ to the fiber loop length $z=L$ ), $\tau$ being the fast time in the frame traveling at group velocity of light. Equation (2) accounts for the boundary conditions (i.e., the interferometric and dissipative nature of the system) describing how the field at the beginning of the roundtrip $(m+1)$ is related to the one at the end of the roundtrip $(m)$ and to the injected driving field $E_{i n}$. Here $\rho$ and $\theta$ are the reflection and transmission coefficients of the input/output coupler such that $\rho^{2}+\theta^{2}=1, \phi_{0}$ is the phase accumulated by the intracavity field during one roundtrip of duration $t_{R}$ (length $L$ ). Moreover $\alpha_{f}$ is 
the power loss coefficient associated with the propagation (linear absorption and splice loss), $\beta_{2}$ the group velocity dispersion which in our case is a function of the position in the fiber loop $(z)$ and $\gamma$ the nonlinear coefficient of the fiber. The finesse (which describes how efficiently light is confined inside the cavity) can be calculated as $F=\pi / \alpha$ where $\alpha=\theta^{2} / 2+\alpha_{f} L$ describes the overall losses of the cavity. Also, it is convenient to introduce a parameter $\Delta=\delta_{0} / \alpha$, dubbed the "normalized detuning", which relates to the phase mismatch between the pump and the nearest cavity resonance through $\phi_{0}=2 k \pi-\delta_{0}$ with $k$ integer. The system is known to be bistable for $\Delta>\sqrt{3}$ [24], which is the regime investigated here.
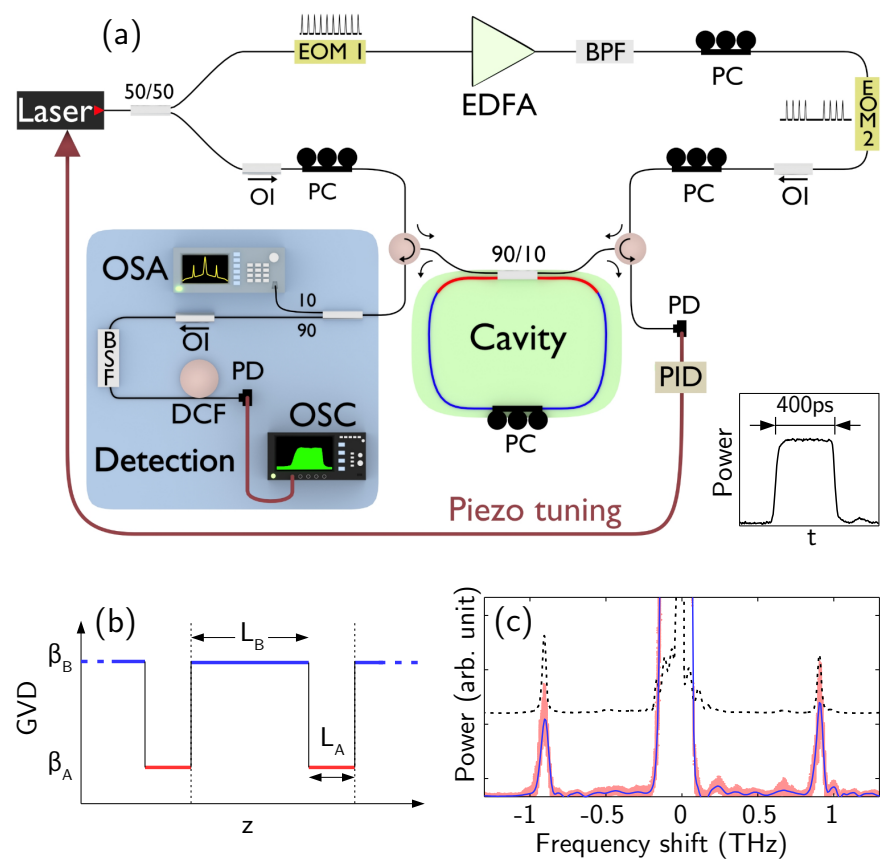

Fig. 1. (a) Experimental setup for the recording of both averaged (OSA) and roundtrip-to-roundtrip (OSC) spectra. Inset: profile of the pump pulses. (b) Longitudinal dispersion profile of the resonator. Two fibers of length $L_{A}$ and $L_{B}$ and GVD $\beta_{A}$ and $\beta_{B}$ are spliced to form a ring. Here $L_{A}=1.8 \mathrm{~m}$; $L_{B}=47.5 \mathrm{~m} ; \beta_{A}=-20.6 \mathrm{ps}^{2} / \mathrm{km} ; \beta_{B}=2 \mathrm{ps}^{2} / \mathrm{km}$ $\left(\bar{\beta}=1.2 \mathrm{ps}^{2} / \mathrm{km}\right)$. (c) Spectrum recorded at the output of the cavity. A trace from a standard optical spectrum analyzer is in dashed black line, 1500 consecutive traces from the DFT technique are in light red with the corresponding average spectrum superimposed in blue. The spectrum from the OSA is upshifted for the sake of clarity. EOM: electro-optic modulator; EDFA: erbium doped fiber amplifier; BPF: band-pass filter; OI: optical isolator; PC: polarization controller; PD: photodetector; PID: proportional-integral-derivative controller; BSF: band-stop filter; DCF: dispersion compensating fiber; OSA: optical spectrum analyser; OSC: oscilloscope.

To investigate the onset of Turing and Faraday instabilities as well as the transition between both regimes in such a fiber ring cavity we used the experimental setup depicted in Fig. 1(a). It is essentially the same as the one described in Ref. [15]. The output of a continuous wave (CW) laser at $1550 \mathrm{~nm}$ is chopped via an electro-optic modulator (EOM 1) into a train of square pulses of $\approx 400$ ps duration (see inset in Fig. 1) at a repetition rate that is finely tuned to fit the free spectral range (FSR) of the cavity. In other words, there is only one pulse circulating inside the cavity at any time. The use of short pulses allows us to access high peak power while mitigating Brillouin scattering. Pulses are amplified by an erbium doped fiber amplifier (EDFA) and a band-pass filter (BPF) removes the amplified spontaneous emission (ASE) in excess. A second EOM (EOM 2) is used to create either periodic bursts of pump pulses (see Fig. 2(a)) or a two-level pump pattern (see Fig. 4(a)). Optical circulators placed before each input of the cavity allow us both to use a single coupler for input and output and to use independently the two directions of propagation inside the resonator. On the right side, the pump pulses are launched inside the fiber ring and circulate in the anti-clockwise direction. This beam is referred to as the nonlinear beam. A fraction of the power of the pump laser is launched in the opposite direction inside the cavity and is used as a reference (linear beam) to actively stabilize the system (using a proportional-integral-derivative (PID) feedback loop which finely tunes the pump wavelength) in order to operate at a fix detuning. The output of the nonlinear beam (which consists of $10 \%$ of the intracavity power that exits the resonator on top of $90 \%$ of the pump reflected by the coupler) enters the detection stage highlighted in light blue. This detection stage allows the simultaneous observation of the output spectrum by means of a standard optical spectrum analyser (OSA) and a fast oscilloscope (OSC) coupled to a dispersion compensating fiber (DCF) using the real-time spectroscopy technique described below. These two techniques give complementary informations during any run of the experiment. Indeed the OSA gives at any time informations concerning the state of the system (i.e., which regime of instability is excited in our case) but fails to reveal the dynamics due to its sweeping time which is in the order of the second, thus spanning a few millions of roundtrips. On the other hand, real-time spectroscopy allows the recording of spectra spanning a few $\mathrm{THz}$ at the characteristic repetition rate of $4.14 \mathrm{MHz}$ of our cavity, though it requires numerical post-processing.

The real time technique implemented here is the DFT, which basically consists on the stretching of the temporal pulses by a highly and purely dispersive element. Note that sufficient stretching is all the easier achieved when the pump pulses are short. In our setup the frequency-to-time conversion is realized by a DCF with a coefficient $\beta_{2}^{D C F} \times L$ of $888 \mathrm{ps}^{2}$. The time-tofrequency mapping is then easily obtained at first order by the relation $T(\omega)=\beta_{2}^{D C F} \times L \times\left(\omega-\omega_{0}\right)$, where $\omega_{0}$ is the angular frequency of the pump. We use a fast photodiode and a $6 \mathrm{GHz}$ oscilloscope for detection and we estimate the spectral resolution to be $\approx 30 \mathrm{GHz}$ [25]. In order to avoid saturation of the photodetector and to be able to reveal weak MI sidelobes, the pump is filtered out using a notch filter (BSF) centered at the pump wavelength.

The fiber cavity (highlighted in green in Fig. 1(a)) is composed of $1.8 \mathrm{~m}$ of standard SMF-28 $\left(\beta_{2}=-20.6 \mathrm{ps}^{2} / \mathrm{km}\right)$ and $47.5 \mathrm{~m}$ of $\operatorname{DSF}\left(\beta_{2}=2 \mathrm{ps}^{2} / \mathrm{km}\right)$, hence exhibiting normal average dispersion. The average nonlinear coefficient is $\gamma=5.5 / \mathrm{W} / \mathrm{km}$. We checked numerically that a realistic modulation of this coefficient does not play a significant role in the dynamics of the cavity. The resonator is closed by a $90 / 10$ input/output coupler $\left(\theta^{2}=0.1\right)$ and we measured a finesse of 19 which corresponds to $\alpha=0.165$.

The accuracy of the frequency-to-time-to-frequency conversion has been checked by comparing the results of the DFT technique to an OSA trace. Driving the resonator with a pulse train of peak power $13 \mathrm{~W}$ and normalized detuning $\Delta=9$ leads to the observation of Faraday instability sidebands in the spectrum. 

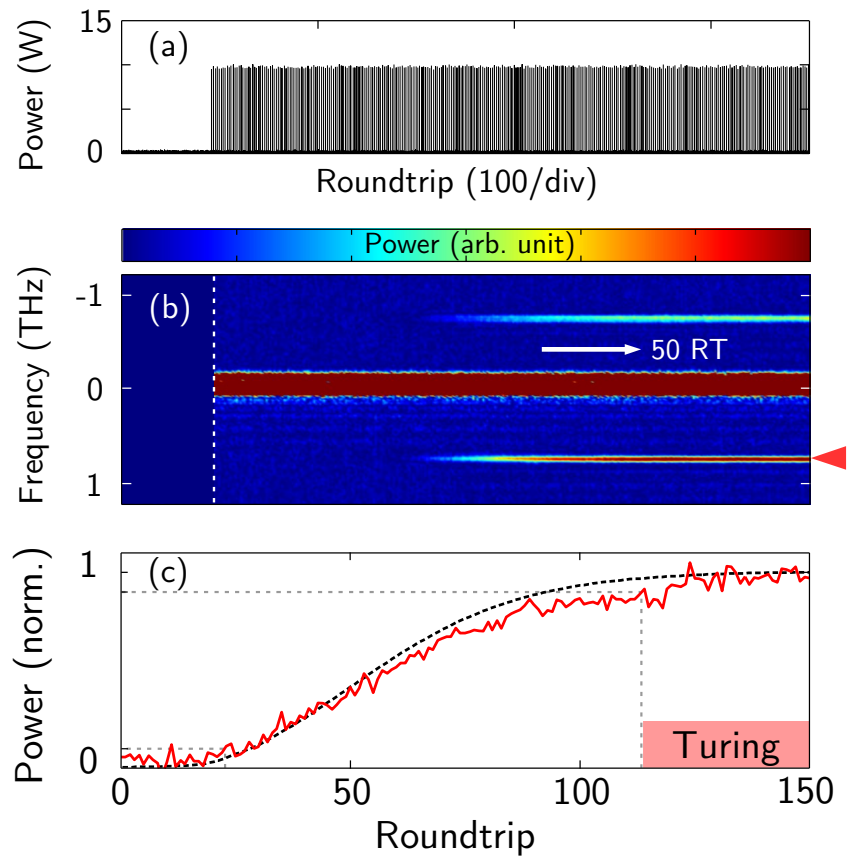

Fig. 2. Input pulse burst resulting in the excitation of Turing instabilities. (b) Experimental roundtrip-to-roundtrip evolution of the spectrum. The dashed line indicates the first input pulse. The Turing sideband position is marked by a red triangle on the right. (c) Evolution of the power contained in the unstable sideband indicated by the colored triangle (see Visualisation 1 for an animated version of the figure). Numerical simulation of this transition is superimposed in dashed black line. Dashed gray lines indicate 10 and $90 \%$ of the rise time.

Figure 1(c) shows an OSA trace along with the superposition of 1500 consecutive spectra acquired using the DFT setup and the corresponding averaged spectrum. A good agreement between the two detection methods is obtained for the range of frequency of interest which validates our setup.

We then proceeded to investigate the onset of the Turing and Faraday sideband growth by launching a burst of pump pulses with constant power. Working at fixed detuning, the nature of the observed instability only depends on the peak power of the pump pulses: in our setting, $10 \mathrm{~W}$ for the Turing branch and $13 \mathrm{~W}$ for the Faraday branch (Fig. 2(a) and 3(a) respectively). The normalised detuning was set henceforth to $\Delta=9$, which is large enough to allow the two regimes of instability to be independently excited [26]. Figure 2(b) and 3(b) show that in both cases a pair of spectral sidebands appear symetrically to the pump after a few roundtrips, at a frequency shift slightly higher for Faraday sidebands, which is in agreement with theory and previous experiments $[9,15]$. Furthermore this allows us to unambiguously identify the observed regime of instability. The growth of the power of the unstable modes eventually saturates as the system reaches a stable attractor of the dynamics [5]. This is clearly illustrated by Fig. 2(c) and 3(c), which showcase the temporal evolution of the power content of the high-frequency sideband in the two regimes. By comparing Fig. 2(c) and 3(c), one clearly notice that the transition to the stable Turing regime appears to be slower than the transition to the Faraday regime. A simple and intuitive explanation lies on the difference of parametric gain between the two regimes of instability. Indeed, we
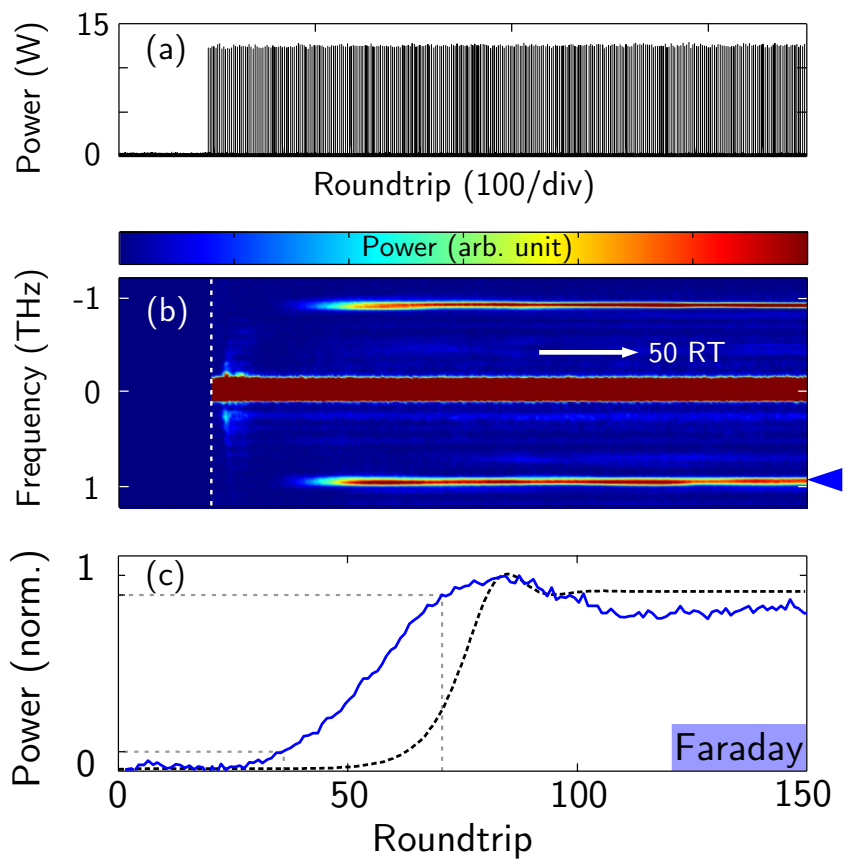

Fig. 3. Same as in Fig. 2 for the case of the excitation of the Faraday instability (see Visualisation 2 for an animated version of the figure).

verified that the parametric gain (estimated from [9] and the supplemental material of [15]) is more than two times higher in the Faraday case compared to the Turing case. On the other hand, the rise time of the Faraday mode is nearly two times shorter than the one of the Turing mode, as can be seen in Fig. 2(c) and Fig. 3(c). This supports the argument that the difference in the dynamics between these two regimes is essentially driven by the strength of the parametric gain.

In order to compare with the experimental recordings, we also conducted numerical simulations based on the the full cavity map [Equations (1) and (2)] including weak monochromatic seeds at the most unstable Turing and Faraday frequencies. The outcome is displayed as dashed black lines in Fig. 2(c) and 3(c). A good qualitative agreement is obtained, clearly highlighting the faster transition in the case of the Faraday instability.

Besides the emergence of the Faraday unstable branch in addition to the Turing one, the inhomogeneous dispersion profile allows the transition between these instability regimes by simply tuning the input power while keeping the detuning fixed. This power dependence has been observed in the stationary regime through the abrupt change of unstable frequency associated to the two instabilities [15]. Here we address the question of the transition between those instabilities by recording the real-time evolution of the cavity output spectrum when abruptly tuning the input pump power from a level corresponding to the Turing regime to a higher one which corresponds to the Faraday regime.

Figure 4(b) shows the evolution of the output spectrum corresponding to the pump power evolution of Fig. 4(a). The abrupt power step is preceded by a large number of pump pulses of $10 \mathrm{~W}$ peak power (not shown here) to ensure that a stable Turing state is reached. Once again, the different regimes of instability can be identified owing to their different characteristic frequencies. Figure 5 (a) showcases the evolution of the normalized 

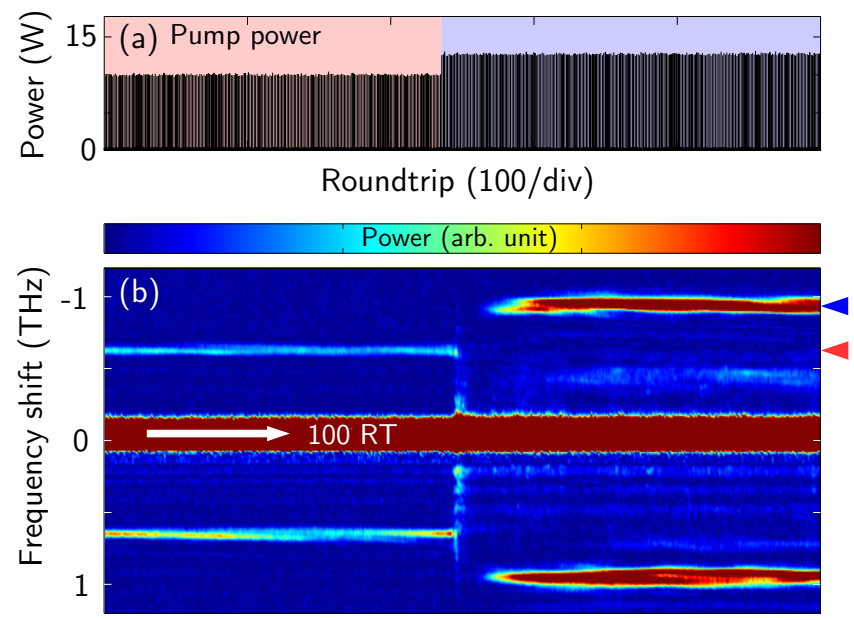

Fig. 4. (a) Input pulse pattern exhibiting an abrupt increase of the pump power. The two levels correspond to peak pump powers of 10 and $13 \mathrm{~W}$. (b) Corresponding experimental evolution of the cavity output spectrum. Turing (Faraday) sideband's position is marked by a red (blue) triangle on the right.
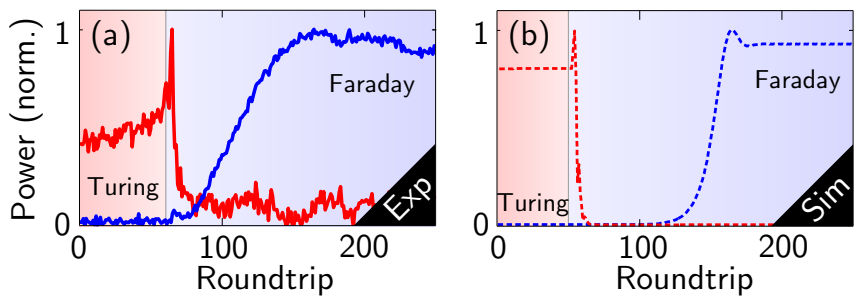

Fig. 5. (a) Experimental evolution of the powers of the Turing and Faraday modes during the "Turing-to-Faraday" transition. (b) Corresponding numerical simulations. Red (blue) lines refer to the Turing (Faraday) mode. The input power switch is indicated by gray vertical lines and a change of background color. See Visualisation 3 for an animated version of the figure.

powers of Turing and Faraday modes during the "Turing-toFaraday" transition. Starting from the stationary state of the Turing mode, the abrupt increase of the input power triggers the transition characterised by a sudden boost of the power of low frequency components before the power of the Turing mode falls to nearly zero. This is rapidly followed by the growth and saturation of the power of the Faraday mode in a fashion very similar to Fig. 3(c). The corresponding simulations shown in Fig. 5(b) accurately reproduce this behaviour. Conversely, we point out that the bistable nature of the system and the underpinning hysteresis (demonstrated in Ref. [15]) can result in a more complex dynamics for the "Faraday-to-Turing" transition, which extends beyond the simple tuning of the input power that we applied here. We thus do not address this case in this letter.

In summary, we recorded the real-time evolution of the optical spectrum at the output of a longitudinally modulated passive fiber ring cavity. Such configuration exhibits two different regimes of instability which can be excited independently depending on the input pump power. Besides sharing some features in common such as the growth and saturation of the power of the unstable band, these regimes differ from one another by their dynamical behaviour. The transition between the two regimes is also investigated and reveals a complex dynamics during which the system switches from one mode to the other without any evidence for their coexistence.

This work has been partially supported by the Agence Nationale de la Recherche through the NoAWE (ANR-14- ACHN0014) and TOPWAVE (ANR-13-JS04-0004) projects, the LABEX CEMPI (ANR-11-LABX-0007) and the Equipex Flux (ANR-11EQPX-0017), as well as by the Ministry of Higher Education and Research, Nord-Pas de Calais Regional Council and European Regional Development Fund (ERDF) through the Contrat de Projets Etat-Region (CPER Photonics for Society P4S) and by IRCICA, USR 3380, CNRS-Univ, F-59000 Lille, France (http://www.ircica.univ-lille1.fr). S. T. acknowledges fund PRIN (2012BFNWZ2).

\section{REFERENCES}

1. V. E. Zakharov and L. A. Ostrovsky, Physica D: Nonlinear Phenomena 238, 540 (2009).

2. L. A. Lugiato and R. Lefever, Phys. Rev. Lett. 58, 2209 (1987).

3. A. M. Turing, Philosophical Transactions of the Royal Society of London B: Biological Sciences 237, 37 (1952).

4. M. Nakazawa, K. Suzuki, and H. A. Haus, Phys. Rev. A 38, 5193 (1988).

5. M. Haelterman, S. Trillo, and S. Wabnitz, Opt. Lett. 17, 745 (1992).

6. M. Haelterman, S. Trillo, and S. Wabnitz, Optics Communications 93, 343 (1992).

7. S. Coen and M. Haelterman, Phys. Rev. Lett. 79, 4139 (1997).

8. S. Coen and M. Haelterman, Opt. Lett. 26, 39 (2001).

9. M. Conforti, A. Mussot, A. Kudlinski, and S. Trillo, Opt. Lett. 39, 4200 (2014).

10. M. Conforti, F. Copie, A. Mussot, A. Kudlinski, and S. Trillo, Opt. Lett., OL 41, 5027 (2016).

11. M. Faraday, Philosophical Transactions of the Royal Society of London 121, 299 (1831).

12. K. Staliunas, C. Hang, and V. V. Konotop, Phys. Rev. A 88, 023846 (2013).

13. N. Tarasov, A. M. Perego, D. V. Churkin, K. Staliunas, and S. K. Turitsyn, Nature Communications 7, 12441 (2016).

14. P. Engels, C. Atherton, and M. A. Hoefer, Phys. Rev. Lett. 98, 095301 (2007).

15. F. Copie, M. Conforti, A. Kudlinski, A. Mussot, and S. Trillo, Phys. Rev. Lett. 116, 143901 (2016).

16. D. R. Solli, G. Herink, B. Jalali, and C. Ropers, Nat Photon 6, 463 (2012).

17. X. Wang, D. Bigourd, A. Kudlinski, K. K. Y. Wong, M. Douay, L. Bigot, A. Lerouge, Y. Quiquempois, and A. Mussot, Opt. Lett. 39, 1881 (2014).

18. B. Wetzel, A. Stefani, L. Larger, P. A. Lacourt, J. M. Merolla, T. Sylvestre, A. Kudlinski, A. Mussot, G. Genty, F. Dias, and J. M. Dudley, Scientific Reports 2, 882 (2012).

19. M. J. Schmidberger, P. Hosseini, D. Novoa, A. Stefani, P. S. Russell, and N. Joly, "Spectrally resolved shot-to-shot nonlinear dynamics of a passive PCF ring cavity," (OSA, 2014), p. SW1I.2.

20. A. F. J. Runge, N. G. R. Broderick, and M. Erkintalo, Journal of the Optical Society of America B 33, 46 (2016).

21. J. Hammer, P. Hosseini, C. R. Menyuk, P. St.J. Russell, and N. Y. Joly, Optics Letters 41, 4641 (2016).

22. G. Herink, B. Jalali, C. Ropers, and D. R. Solli, Nature Photonics 10, 321 (2016).

23. K. Ikeda, Optics Communications 30, 257 (1979).

24. M. Haelterman, S. Trillo, and S. Wabnitz, Opt. Commun. 91, 401 (1992).

25. K. Goda and B. Jalali, Nat Photon 7, 102 (2013).

26. S. Coen, M. Haelterman, P. Emplit, L. Delage, L. M. Simohamed, and F. Reynaud, J. Opt. B: Quantum Semiclass. Opt. 1, 36 (1999). 


\section{FULL REFERENCES}

1. V. E. Zakharov and L. A. Ostrovsky, "Modulation instability: The beginning," Physica D: Nonlinear Phenomena 238, 540-548 (2009).

2. L. A. Lugiato and R. Lefever, "Spatial Dissipative Structures in Passive Optical Systems," Phys. Rev. Lett. 58, 2209 (1987).

3. A. M. Turing, "The Chemical Basis of Morphogenesis," Philosophical Transactions of the Royal Society of London B: Biological Sciences 237, 37 (1952).

4. M. Nakazawa, K. Suzuki, and H. A. Haus, "Modulational instability oscillation in nonlinear dispersive ring cavity," Phys. Rev. A 38, 51935196 (1988).

5. M. Haelterman, S. Trillo, and S. Wabnitz, "Additive-modulationinstability ring laser in the normal dispersion regime of a fiber," Opt. Lett. 17, 745-747 (1992).

6. M. Haelterman, S. Trillo, and S. Wabnitz, "Low dimensional modulational chaos in diffractive nonlinear cavities," Optics Communications 93, 343-349 (1992).

7. S. Coen and M. Haelterman, "Modulational Instability Induced by Cavity Boundary Conditions in a Normally Dispersive Optical Fiber," Phys. Rev. Lett. 79, 4139-4142 (1997).

8. S. Coen and M. Haelterman, "Continuous-wave ultrahigh-repetitionrate pulse-train generation through modulational instability in a passive fiber cavity," Opt. Lett. 26, 39-41 (2001).

9. M. Conforti, A. Mussot, A. Kudlinski, and S. Trillo, "Modulational instability in dispersion oscillating fiber ring cavities," Opt. Lett. 39, 4200-4203 (2014).

10. M. Conforti, F. Copie, A. Mussot, A. Kudlinski, and S. Trillo, "Parametric instabilities in modulated fiber ring cavities," Opt. Lett., OL 41, 50275030 (2016).

11. M. Faraday, "On a Peculiar Class of Acoustical Figures; and on Certain Forms Assumed by Groups of Particles upon Vibrating Elastic Surfaces," Philosophical Transactions of the Royal Society of London 121, 299 (1831).

12. K. Staliunas, C. Hang, and V. V. Konotop, "Parametric patterns in optical fiber ring nonlinear resonators," Phys. Rev. A 88, 023846 (2013).

13. N. Tarasov, A. M. Perego, D. V. Churkin, K. Staliunas, and S. K. Turitsyn, "Mode-locking via dissipative Faraday instability," Nature Communications 7, 12441 (2016).

14. P. Engels, C. Atherton, and M. A. Hoefer, "Observation of Faraday Waves in a Bose-Einstein Condensate," Phys. Rev. Lett. 98, 095301 (2007).

15. F. Copie, M. Conforti, A. Kudlinski, A. Mussot, and S. Trillo, "Competing Turing and Faraday Instabilities in Longitudinally Modulated Passive Resonators," Phys. Rev. Lett. 116, 143901 (2016).

16. D. R. Solli, G. Herink, B. Jalali, and C. Ropers, "Fluctuations and correlations in modulation instability" Nat Photon 6, 463 (2012).

17. X. Wang, D. Bigourd, A. Kudlinski, K. K. Y. Wong, M. Douay, L. Bigot, A. Lerouge, Y. Quiquempois, and A. Mussot, "Correlation between multiple modulation instability side lobes in dispersion oscillating fiber"Opt. Lett. 39, 1881 (2014).

18. B. Wetzel, A. Stefani, L. Larger, P. A. Lacourt, J. M. Merolla, T. Sylvestre, A. Kudlinski, A. Mussot, G. Genty, F. Dias, and J. M. Dudley, "Realtime full bandwidth measurement of spectral noise in supercontinuum generation" Scientific Reports 2, 882 (2012).

19. M. J. Schmidberger, P. Hosseini, D. Novoa, A. Stefani, P. S. Russell, and N. Joly, "Spectrally resolved shot-to-shot nonlinear dynamics of a passive PCF ring cavity," (OSA, 2014), p. SW1I.2.

20. A. F. J. Runge, N. G. R. Broderick, and M. Erkintalo, "Dynamics of soliton explosions in passively mode-locked fiber lasers," Journal of the Optical Society of America B 33, 46 (2016).

21. J. Hammer, P. Hosseini, C. R. Menyuk, P. St.J. Russell, and N. Y. Joly, "Single-shot reconstruction of spectral amplitude and phase in a fiber ring cavity at a $80 \mathrm{MHz}$ repetition rate," Optics Letters 41, 4641 (2016).

22. G. Herink, B. Jalali, C. Ropers, and D. R. Solli, "Resolving the build-up of femtosecond mode-locking with single-shot spectroscopy at $90 \mathrm{MHz}$ frame rate,"Nature Photonics 10, 321 (2016).

23. K. Ikeda, "Multiple-valued stationary state and its instability of the transmitted light by a ring cavity system," Optics Communications $\mathbf{3 0}$,
257-261 (1979).

24. M. Haelterman, S. Trillo, and S. Wabnitz, "Dissipative modulation instability in a nonlinear dispersive ring cavity," Opt. Commun. 91, 401 (1992).

25. K. Goda and B. Jalali, "Dispersive Fourier transformation for fast continuous single-shot measurements," Nat Photon 7, 102 (2013).

26. S. Coen, M. Haelterman, P. Emplit, L. Delage, L. M. Simohamed, and F. Reynaud, "Bistable switching induced by modulational instability in a normally dispersive all-fibre ring cavity," J. Opt. B: Quantum Semiclass. Opt. 1, 36 (1999). 